\title{
Cost-Utility Analysis of Dapagliflozin Compared to Sulfonylureas for Type 2 Diabetes as Second- Line Treatment in Indian Healthcare Payer's Perspective
}

\author{
Bhavani Shankara Bagepally,2 \\ Usa Chaikledkaew ${ }^{1,3}$ \\ Sitaporn Youngkong, ${ }^{1,3}$ \\ Thunyarat Anothaisintawee $e^{1,4}$ \\ Montarat Thavorncharoensap 1,3 \\ Charungthai Dejthevaporn ${ }^{5}$ \\ Ammarin Thakkinstian ${ }^{1,6}$ \\ 'Mahidol University Health Technology \\ Assessment (MUHTA) Graduate \\ Program, Mahidol University, Bangkok, \\ Thailand; ${ }^{2}$ Department of Non- \\ Communicable Diseases, ICMR-National \\ Institute of Epidemiology, Chennai, India; \\ ${ }^{3}$ Social and Administrative Pharmacy \\ Division, Department of Pharmacy, \\ Faculty of Pharmacy, Mahidol University, \\ Bangkok, Thailand; ${ }^{4}$ Department of \\ Family Medicine, Faculty of Medicine \\ Ramathibodi Hospital, Mahidol \\ University, Bangkok, Thailand; \\ ${ }^{5}$ Department of Medicine, Faculty of \\ Medicine Ramathibodi Hospital, Bangkok, \\ Thailand; ${ }^{6}$ Department of Clinical \\ Epidemiology and Biostatistics, Faculty of \\ Medicine Ramathibodi Hospital, Mahidol \\ University, Bangkok, Thailand
}

Background: Type 2 diabetes mellitus (T2DM) is a leading health issue, causing economic burden in India. Pharmacotherapy is a major cost driver in diabetic care usually funded through out of pocket expenditure; however, there has been a very limited economic evaluation evidence to guide the choice of diabetes pharmacotherapy in India. Therefore, this study aims to evaluate the long-term cost-effectiveness of dapagliflozin (sodium glucose transporter 2 inhibitor) compared to commonly used sulfonylureas as second-line drugs in Indian patients with T2DM.

Methods: Cost-utility analysis was employed to estimate the costs and health outcomes using a Markov model with 1-year cycle length during a lifetime horizon based on an Indian payer's perspective. A treatment pathway with dapagliflozin as second-line therapy was compared to sulfonylureas after failure of initial metformin therapy. Clinical and cost data were collected from literature reviews and available secondary data sources. Both costs and outcomes were discounted at a 3\% annual discount rate. The results were presented as the incremental cost-effectiveness ratio (ICER). One-way and probabilistic sensitivity analyses were performed to test parameter uncertainties.

Results: Compared to sulfonylurea, dapagliflozin was estimated to incur an additional cost of ₹182,632 (US\$2,446) with an expected 3.49 life years (LY) or 1.72 quality adjusted life years (QALY) gained, resulting in an ICER of ₹52,270 (US\$699) per LY gained, or ₹106,133 (US\$1,421) per QALY gained. Uncertainty analyses showed that the ICER values were not sensitive to changes in most parameters.

Conclusion: Dapagliflozin would be cost-effective compared to sulfonylureas as the second line added to metformin for T2DM patients based on an Indian payer's perspective.

Keywords: economic evolution, cost-effectiveness, sodium glucose transporter 2 inhibitor, type 2 diabetes, India, glibenclamide, gliclazide

\section{Introduction}

Diabetes is a leading public health burden in the world with approximately 431 million people ${ }^{1}$ and type 2 diabetes (T2DM) is the most common cause of diabetes $(90 \%)$. $^{2}$ Published literature indicate that nearly half (range 24.1-75.1\%) of diabetes cases among adults were undiagnosed (about 174.8 million), and 72 million were found in India, resulting in $8.8 \%$ of global burden. ${ }^{3-5}$ T2DM is one of the leading causes of mortality and morbidity in India, ${ }^{6}$ as it is the second
Correspondence: Usa Chaikledkaew Social Administrative Pharmacy Division, Department of Pharmacy, Faculty of Pharmacy, Mahidol University, 447 SriAyudhaya Road, Phayathai, Ratchathewi, Bangkok, 10400, Thailand

Tel +662-644-8677 ext 53। 7

Fax +662-644-8694

Email usa.chi@mahidol.ac.th 
most common individual cause of death, accounting for more than $5 \%$ of all deaths. The all age death rate was markedly raised from $111 \%$ to $150 \%$ and the agestandardized death rate from $48 \%$ to $79 \%$ from 1990 to 2016. Furthermore, it is the thirteenth leading cause of total disability adjusted life years (DALY) ${ }^{7}$ with the highest progression rate $(80 \%)$ for all-age and agestandardized DALY $^{6}$ in India. A recent systematic review also highlighted the high economic burden of diabetes among individuals and households in India. ${ }^{8}$ The International Diabetes Federation (IDF) estimated that diabetes was attributed to approximately 5 million deaths, resulting in a global healthcare expenditure of US $\$ 850$ billion in the year $2017 .^{2}$ As the world's diabetes burden has been continuously growing, healthcare expenditures related to $\mathrm{T} 2 \mathrm{DM}$ reached $6.4 \%$ in worldwide. ${ }^{9,10}$

T2DM is mainly managed with lifestyle medications along with pharmacotherapy with anti-diabetic drugs. ${ }^{11}$ Selection of pharmacotherapy for T2DM patients is often complex due to a number of factors such as unintended sequelae, ie, hypoglycemia, weight changes, side-effects that can have a significant impact on patients' adherence, and quality-of-life. ${ }^{11}$ It is recommended that metformin should be a first-line drug followed by dual and triple antidiabetic drugs successively added depending on the patients' glycemic control according to standard guidelines. ${ }^{11}$ Based on the 2018 American Diabetes Association guidelines, sulfonylureas are commonly prescribed as second-line treatment with metformin when T2DM patients are not at risk for cardiovascular diseases due to the concerns regarding long-term cardiac safety and the risk of hypoglycemia. ${ }^{12}$ In addition, it is recommended that sodium-glucose transport inhibitors (SGLT2) and glucagon-like peptide 1 receptor agonists (GLP-1) are much better alternatives as second-line treatment, as these drugs have shown additional improvement in glucose control and reduction cardiovascular events in T2DM with atherosclerotic cardiovascular diseases. ${ }^{13}$

Gliflozin class or SGLT2 can reduce glucose reabsorption in the proximal tubule of the kidney, leading to urinary glucose excretion and osmotic diuresis. Dapagliflozin is the first SGLT2 inhibitor approved for glycemic control in T2DM anywhere in the world, including India. Currently, the price of dapagliflozin is more expensive than sulfonylureas, and this can increase the cost of T2DM medications, ${ }^{14}$ of which $80 \%$ are mainly paid out-of-pocket by the patients and their families in
India. ${ }^{15}$ Therefore, economic evaluation of dapagliflozin is required to confirm whether it is cost-effective to be used as second-line therapy for T2DM patients in India. Based on our literature search, 13 economic evaluation studies were conducted in developed countries, but none were performed in low- and middle-income countries such as India ${ }^{16}$. Therefore, our study aimed to investigate the cost-utility of dapagliflozin compared to sulfonylureas as second-line therapy in T2DM patients based on an Indian payer's perspective. The results of our study could be used as evidence-based information for physicians as to whether dapagliflozin would be cost-effective to be prescribed as second-line therapy compared to sulfonylureas for T2DM patients in India.

\section{Methods}

A cost-utility analysis (CUA) using a Markov model was conducted to compare the costs and health outcomes of dapagliflozin $(10 \mathrm{mg} /$ day $)$ with a usual treatment with sulfonylureas over a lifetime period. The study was performed based on an Indian payer's perspective, as $80 \%$ of healthcare costs are paid by patients' out of pocket in India. Target populations were newly diagnosed Indian T2DM patients aged 30 years, ie, the age at diabetic screening under the National Program for Prevention and Control of Cancer, Diabetes, Cardiovascular Diseases and Stroke in India. ${ }^{16}$ We compared two different treatment pathways. First, for a current practice in India, T2DM patients received metformin monotherapy, and, if it failed, sulfonylureas were added as second-line therapy, and finally insulin was added due to the second-line treatment failure. Second, for a new intervention, T2DM patients were prescribed with metformin monotherapy as first line treatment and received dual therapy, ie, metformin plus dapagliflozin due to first line treatment failure, finally received triple therapy, ie, metformin, dapagliflozin plus insulin when dual therapy failed. The treatment failure is defined as T2DM patients unable to maintain glycemic control (HbA1C $<7 \%$ ) according to the ADA guidelines. ${ }^{12}$ An annual discounting rate of $3 \%$ was applied to both costs and outcomes. ${ }^{17}$ The incremental cost-effectiveness ratio (ICER) was calculated by a difference in costs (Indian rupees, ₹) divided by a difference in life years (LY) or quality-adjusted life years (QALY) gained between two aforementioned alternatives. As there is no standard threshold in India, we referred to the recommendation from the World Health Organization (WHO), ie, less than one gross domestic product (GDP) per capita per QALY gained 
considered as highly cost-effective, 1-3times GDP as costeffective and more than 3-times GDP as not cost-effective. In India, one time GDP in the year 2019 equal to INR $₹ 1,56,798^{19}$ was applied as the willingness to pay (WTP) threshold. ${ }^{17}$ In addition, the incremental net benefit (INB) was calculated as the multiplication of the WTP threshold and incremental QALY minus incremental cost.

\section{Model Overview}

Figure 1 illustrates the Markov model simulating T2DM treatment pathways. The model consists of four health states, as follows: 1) metformin monotherapy, 2) dual therapy, ie, metformin plus second-line treatment of either sulfonylureas or dapagliflozin if first line treatment failed, 3) triple therapy, ie, metformin plus second line plus insulin as a third line drug, if second-line treatment failed, and 4) death. The arrows represent the transitional probabilities of moving from one health state to another. The model was applied to estimate costs and outcomes during a lifetime period with a 1-year cycle length using Microsoft Office Excel 2016 (Microsoft Corp., Redmond, WA).

The model started with T2DM patients initially treated with metformin monotherapy. If first line treatment failed, second-line therapy of either dapagliflozin or sulfonylureas was added to metformin for controlling the blood glucose level. ${ }^{12}$ T2DM patients who were on metformin monotherapy could remain in the same health state or move to dual therapy due to first line treatment failure. In the dual therapy state, T2DM patients could remain in the same state or move to triple therapy, where insulin was added, owing to second-line treatment failure. T2DM patients in any of the three health states could move to the death state. We considered the costs and outcomes related to complications such as hypoglycemia, myocardial infarction, heart failure, stroke and genital infection among T2DM patients receiving dual or triple therapy. These complications were selected based on the recent publication reporting the common complications among diabetics in India and the availability of relevant model input data. ${ }^{20}$ For hypoglycemia, we proportionated symptomatic hypoglycemia (symptomatic with no need of medical attention) and severe (requiring medical attention) for utility calculation. Only severe hypoglycemia costs were considered. It was assumed that the cohort did not have any baseline comorbidities.

\section{Model Inputted Parameters Clinical and Utility Data}

Table 1 demonstrates all parameters used in the Markov model. The transitional probabilities of dual therapy for dapagliflozin and sulfonylureas were taken from a randomized controlled trial study which compared directly between dapagliflozin and sulfonylurea. ${ }^{20}$ Moreover, the probabilities of complications in each state were retrieved from published literature. Data on age standardized death rate were obtained from an open government data forum of India's website (https://data.gov.in/ catalog/estimated-age-specific-death-rates-sex). Due to a limited availability of head-to-head comparison between dapagliflozin and sulfonylureas in India, we obtained the efficacy data from a study by Nauck et $\mathrm{al}^{21}$ which compared dapagliflozin and glipizide. As a result, it was assumed that drug class effects across sulfonylureas were similar. Utility decrement values associated with complications were obtained from a systematic literature search. The initial utility value of diabetic patients during the first diagnosis was assumed to be equal to 1 . The utility values calculated by the differences between initial utility and disutilities and all utility values are taken into account for all cycles.

\section{Cost Data}

The costs of commonly prescribed sulfonylureas, ie, glibenclamide and glimepiride tablets, were obtained from the prices of the National Pharmaceutical Pricing Authority with $12 \%$ of goods and service tax. ${ }^{22,23}$ The cost of sulfonylureas was the average cost of glibenclamide and glimepiride per tablet. The costs of dapagliflozin and human insulin $(100 \mathrm{IU} / \mathrm{mL})$ were retrieved from the market prices at the website of pharmacy chain stores in India. ${ }^{24} \mathrm{We}$ assumed that a daily dose for insulin requirement was 30 $\mathrm{IU} / \mathrm{day}^{25}$ for sulfonylureas and $20.5 \mathrm{IU} / \mathrm{day}^{26}$ for dapagliflozin. All costs were expressed in Indian rupees (₹), adjusted to the values in the year 2019 using the consumer price indexes (CPI), converted to the United State dollars (USD) with the exchange rate of ₹74.68 per USD.

\section{Uncertainty Analysis}

Both univariate and probabilistic sensitivity analyses (PSA) using the second order Monte Carlo simulation were performed to handle parameter uncertainties. For a univariate sensitivity analysis, each inputted parameter was varied by $25 \%$ for the upper and lower values. PSA was conducted to investigate the impact of parameter 
uncertainties of all parameters. We assigned beta distribution for transitional probabilities and utility parameters and gamma distribution for cost parameters. Parameter values were randomly drawn from these distributions 1,000 times to estimate the mean costs, LYs, and QALYs. Univariate sensitivity analysis results were presented as a tornado diagram and PSA results were shown as the costeffectiveness plane and cost-effectiveness acceptability curve (CEAC).

\section{Results}

\section{Cost-Effectiveness Analysis}

Table 2 presents the cost-effectiveness results. The total costs, LYs, and QALYs of second-line therapy were ₹292,416 (\$3,915), 158.66 LYs, and 69.64 QALYs for sulfonylureas and ₹475,048 (\$6,361), 162.16 LYs, and 71.36 QALYs for dapagliflozin. Compared to sulfonylureas, dapagliflozin therapy incurred an additional cost of ₹1,82,632 $(\$ 2,445)$, with an additional 3.49 LYs or 1.72 QALYs, resulting in an ICER of ₹52,270 (\$699) per LY gained, or $₹ 1,06,133(\$ 1,421)$ per QALY gained. The corresponding INB calculated from PSA result was ₹2,69,895 $\pm 47,217$ $(\$ 3,614 \pm 632)$. Based on the cost-effectiveness plane (Figure 2), all ICER values were located on the upper-right hand quadrant of the plane, indicating the higher the incremental costs the higher QALYs of dapagliflozin compared to sulfonylureas. Figure 3 presents the cost-effectiveness acceptability curve demonstrating that at the current costeffectiveness threshold or WTP at one time GDP per capita per QALY gained, the probability of being cost-effective for dapagliflozin therapy was $100 \%$.

\section{Uncertainty Analysis}

Figure 4 demonstrates univariate sensitivity analysis as a tornado diagram. The utmost sensitive parameters to influence the ICER values were transitional probabilities of metformin treatment failure, dual therapy failure, and cost of triple therapy. The cost of triple therapy had the highest influence on the ICER change (36.8\%). The values of transitional probabilities inversely influenced the ICER values, ie, with the upper values of metformin failure rates in sulfonylureas, T2DM patients tended to lower the ICER values. On the other hand, the ICER values were negligibly sensitive to the probability of death, hypoglycemic incidences, and utility values. Based on the sensitivity analysis by using the same insulin requirement for both dapagliflozin and sulfonylurea, the ICER (₹101,933) is slightly lowered, suggesting the results are valid even with the same insulin dose requirement.

\section{Discussion}

This is the first study to investigate the cost-utility of a treatment pathway with dapagliflozin as a second-line therapy compared to sulfonylureas for T2DM based on an Indian payer's perspective during lifetime period using a Markov model. The results suggested that dapagliflozin as second-line therapy added to metformin monotherapy would be cost-effective at the WTP per QALY gained in India or one-time GDP per capita referred to the WHO recommendations. Based on the results from CEAC with different WTP level, our study revealed that at the WTP of one-time GDP per capita per QALY gained, dapagliflozin had a $100 \%$ chance of being cost-effective compared to sulfonylureas. In addition, dapagliflozin and sulfonylureas as second-line therapy had a $50 \%$ chance of being costeffective at the WTP per QALY gained of approximately $₹ 69,000$. Likewise, our study results were similar to previous studies comparing the costs and outcomes of dapagliflozin with sulfonylureas as well as dipeptidyl peptidase-4 inhibitors as second-line therapy for T2DM patients in the United Kingdom. ${ }^{27,28}$ Similar to the reports from the Nordic countries, ${ }^{28}$ the total costs and LYs of dapagliflozin were

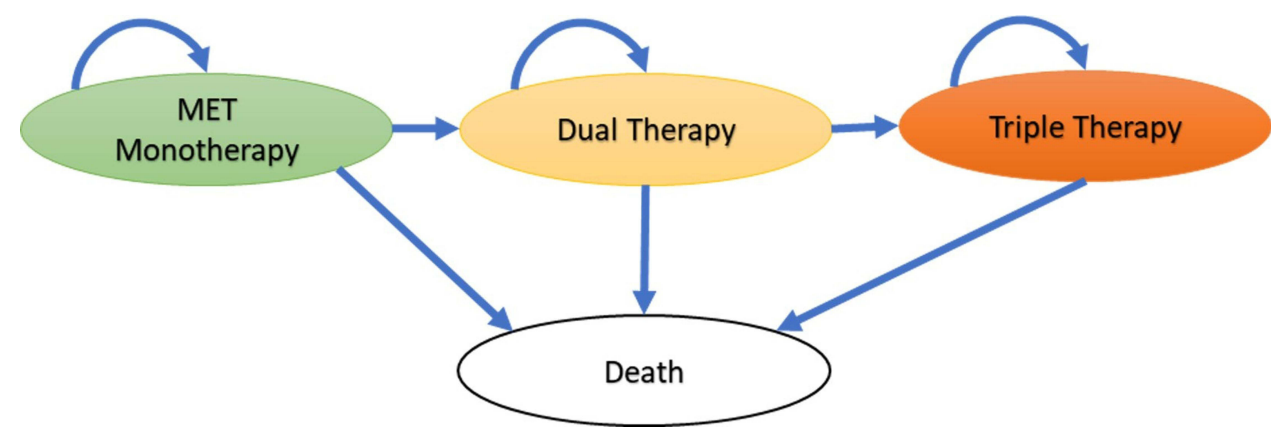

Figure I Schematic diagram of the Markov Model. Author's compilation based on treatment pathways. 
Table I Parameters Used in the Model

\begin{tabular}{|c|c|c|c|}
\hline Probabilities & Mean (SE) & Distribution & Source \\
\hline MET monotherapy failure & $0.043(0.0053)$ & Beta & Kahn et $\mathrm{al}^{32}$ \\
\hline MET+DAPA failure & $0.102(0.0179)$ & Beta & Nauck et al $2014^{21}$ \\
\hline MET+SU failure & $0.156(0.0219)$ & Beta & Nauck et al $2014^{21}$ \\
\hline Death from MET monotherapy & $0.019(0.0004)$ & Beta & Mohan et al $2006^{33}$ \\
\hline MET+DAPA treatment to death & $0.008(0.0028)$ & Beta & Toulis et al $2017^{34}$ \\
\hline$M E T+S U$ treatment to death & $0.012(0.0012)$ & Beta & Varvaki Rados et al $2016^{35}$ \\
\hline $\mathrm{MET}+\mathrm{SU}+$ Ins treatment to death & $0.096(0.0040)$ & Beta & Anyanwagu et al $2016^{36}$ \\
\hline MET+DAPA+Ins treatment to death & $0.008(0.0028)$ & Beta & Toulis et al $2017^{34}$ \\
\hline \multicolumn{4}{|l|}{ Complications } \\
\hline \multicolumn{4}{|l|}{ Hypoglycemia } \\
\hline With MET+SU & $0.1362(0.0051)$ & Beta & Mishriky et al $2015^{37}$ \\
\hline With MET+SU requiring in-hospital treatment & $0.0681(0.0038)$ & Beta & Goke et al $2010^{38}$ \\
\hline With MET+DAPA & $0.0209(0.007 \mathrm{I})$ & Beta & Nauck et $\mathrm{al}^{21}$ \\
\hline With Ins & $0.0100(0.0099)$ & Beta & ORIGIN trial ${ }^{39}$ \\
\hline \multicolumn{4}{|l|}{ Myocardial infarction } \\
\hline With MET & $0.0156(0.0032)$ & Beta & Kahn et al $2006^{32}$ \\
\hline With MET+DAPA & $0.0049(0.0069)$ & Beta & Kosiborod et al $2018^{40}$ \\
\hline With MET+SU & $0.0102(0.0032)$ & Beta & Roumie et al $2014^{41}$ \\
\hline With Ins & $0.0093(0.0096)$ & Beta & ORIGIN trial ${ }^{39}$ \\
\hline \multicolumn{4}{|l|}{ Heart failure } \\
\hline With MET & $0.0033(0.0032)$ & Beta & Kahn et al $2006^{32}$ \\
\hline With MET + DAPA & $0.0060(0.0077)$ & Beta & Kosiborod et al $2018^{40}$ \\
\hline With MET + SU & $0.0196(0.0112)$ & Beta & Gitt et al $2013^{42}$ \\
\hline With Ins & $0.0085(0.0091)$ & Beta & ORIGIN trial $^{39}$ \\
\hline \multicolumn{4}{|l|}{ Stroke } \\
\hline With MET & $0.0032(0.0015)$ & Beta & Kahn et al $2006^{32}$ \\
\hline With MET+DAPA & $0.0045(0.0067)$ & Beta & Kosiborod et al $2018^{40}$ \\
\hline With MET+SU & $0.0119(0.0034)$ & Beta & Roumie et al $2014^{41}$ \\
\hline With Ins & $0.0091(0.0094)$ & Beta & ORIGIN trial ${ }^{38}$ \\
\hline \multicolumn{4}{|l|}{ Other } \\
\hline Genital infection with MET + DAPA & $0.0679(0.0033)$ & Beta & Puckrin et al $2018^{43}$ \\
\hline \multicolumn{4}{|l|}{ Health utility decrement } \\
\hline Myocardial infarction & $-0.0550(0.0061)$ & Beta & Clarke et al $2004^{44}$ \\
\hline Heart failure & $-0.1080(0.031 \mathrm{I})$ & Beta & Clarke et al $2004^{44}$ \\
\hline Stroke & $-0.1640(0.0295)$ & Beta & Clarke et al $2004^{44}$ \\
\hline Severe hypoglycemia & $-0.0142(0.0018)$ & Beta & Currie et al $2006^{45}$ \\
\hline Symptomatic hypoglycemia & $-0.0470(0.0150)$ & Beta & Currie et al $2006^{45}$ \\
\hline Genital infection & $-0.003(0.0001)$ & Beta & Barry et al $1997^{46}$ \\
\hline Insulin injection & $-0.0200(0.0050)$ & Beta & Zhang et al $2012^{47}$ \\
\hline \multicolumn{4}{|l|}{ Costs per year } \\
\hline Cost of MET monotherapy & $₹|2| 1$ & Gamma & NPPO ceiling price 22 \\
\hline Cost of MET+SU & $₹ 6186$ & Gamma & NPPO ceiling price 22 \\
\hline
\end{tabular}


Table I (Continued).

\begin{tabular}{|c|c|c|c|}
\hline Probabilities & Mean (SE) & Distribution & Source \\
\hline Cost of MET+DAPA & $₹ 35,850$ & Gamma & Med-plus ${ }^{24}$ \\
\hline Cost of MET+SU+Ins & $₹ 94,334$ & Gamma & NPPO ceiling price ${ }^{22}$ and Medplus ${ }^{48}$ \\
\hline Cost of MET+DAPA+Ins & $₹ 96,951$ & Gamma & NPPO ceiling price ${ }^{22}$ and Medplus ${ }^{24,48}$ \\
\hline Glibenclamide 4 mg (per tablet) & $₹ 833$ & Gamma & NPPO ceiling price ${ }^{22}$ \\
\hline Glimepiride $4 \mathrm{mg}$ & ₹9116 & Gamma & NPPO ceiling price ${ }^{22}$ \\
\hline Dapagliflozin_10 mg & $₹ 34,638$ & Gamma & Med-plus ${ }^{24}$ \\
\hline Cost of insulin_per_20.5 unit per day & $₹ 58,363$ & Gamma & Medplus ${ }^{48}$ \\
\hline Cost of insulin_per_30 unit per day & $₹ 85,410$ & Gamma & Medplus ${ }^{48}$ \\
\hline Insulin syringe & $₹ 2737$ & Gamma & Medplus ${ }^{48}$ \\
\hline \multicolumn{4}{|l|}{ Costs of Complications } \\
\hline Hypoglycaemia & $₹ 10,903$ & Gamma & Kwon et al $2018^{49}$ \\
\hline Myocardial Infarction & $₹ 551,192$ & Gamma & Gu et al $2016^{50}$ \\
\hline Heart Failure & $₹ 183,304$ & Gamma & Gu et al $2016^{50}$ \\
\hline Stroke & $₹ 300,436$ & Gamma & Kwatra et al 2013 , Walker et al $2017^{15,51}$ \\
\hline Genital infection & $₹ 4112$ & Gamma & Charokopou et al $2015^{27}$ \\
\hline
\end{tabular}

Notes: Author's compilation based on our reviews on published studies. All costs in Indian Rupee (₹) in 2017.

Abbreviations: MET, metformin; SU, sulfonylurea; DAPA, dapagliflozin; Ins, insulin.

higher than those of sulfonylureas. According to the oneway sensitivity analysis results, the main factors influencing the ICER values were the cost of triple therapy and transitional probabilities of metformin monotherapy failure and dual therapy failure. It should be noted that these probabilities were obtained from international countries due to data scarcity in India. Therefore, future studies with clinical efficacy data for these newer anti-diabetic drugs should be generated from an Indian population.

It is significant to address study limitations. First, we assumed that T2DM patients entered the model at age 30 years old and their long-term outcomes were predicted using data obtained from short-term clinical trials in international countries due to the paucity of specific data in India. It should be noteworthy that the results might not reflect on the real situation in India, but our study might provide the best available cost-effectiveness information for physicians as decision-makers to select cost-effective second-line treatment for T2DM patients. Second, microvascular complications such as amputation, blindness, or end state renal disease were not considered, although patients receiving either dapagliflozin or sulfonylureas might have different costs and outcomes related to microvascular complications. $^{30}$ Third, other adverse events associated with dapagliflozin, ie, diabetic ketoacidosis and acute kidney injury (AKI), were not included in the analysis, because the recent systematic review and meta-analysis of the effect of SGLT2 on renal adverse events demonstrated that SGLT2

Table 2 Cost-Effectiveness Analysis Results

\begin{tabular}{|l|c|c|}
\hline Cost-Effectiveness Analysis Results & Sulfonylureas & Dapagliflozin \\
\hline Total costs & $₹ 292,416(\$ 3,915)$ & $₹ 475,048(\$ 6,36 \mathrm{I})$ \\
Total LYs & 158.66 & 162.16 \\
Total QALYs & 69.64 & 71.36 \\
Incremental costs (₹) & & $₹ 182,632(\$ 2,445)$ \\
Incremental LYs & & 3.49 \\
Incremental QALYs & & 1.72 \\
ICER per LY saved (₹ per LY) & & $₹ 52,270(\$ 699)$ \\
ICER per QALY (₹ per QALY) & & $₹ 106,133(\$ 1,421)$ \\
\hline
\end{tabular}

Notes: Author's compilation based on our cost-effectiveness analysis. Exchange rate $=₹ 74.68$ per USD. Abbreviations: LYs, Life years; QALYs, Quality adjusted life years. 


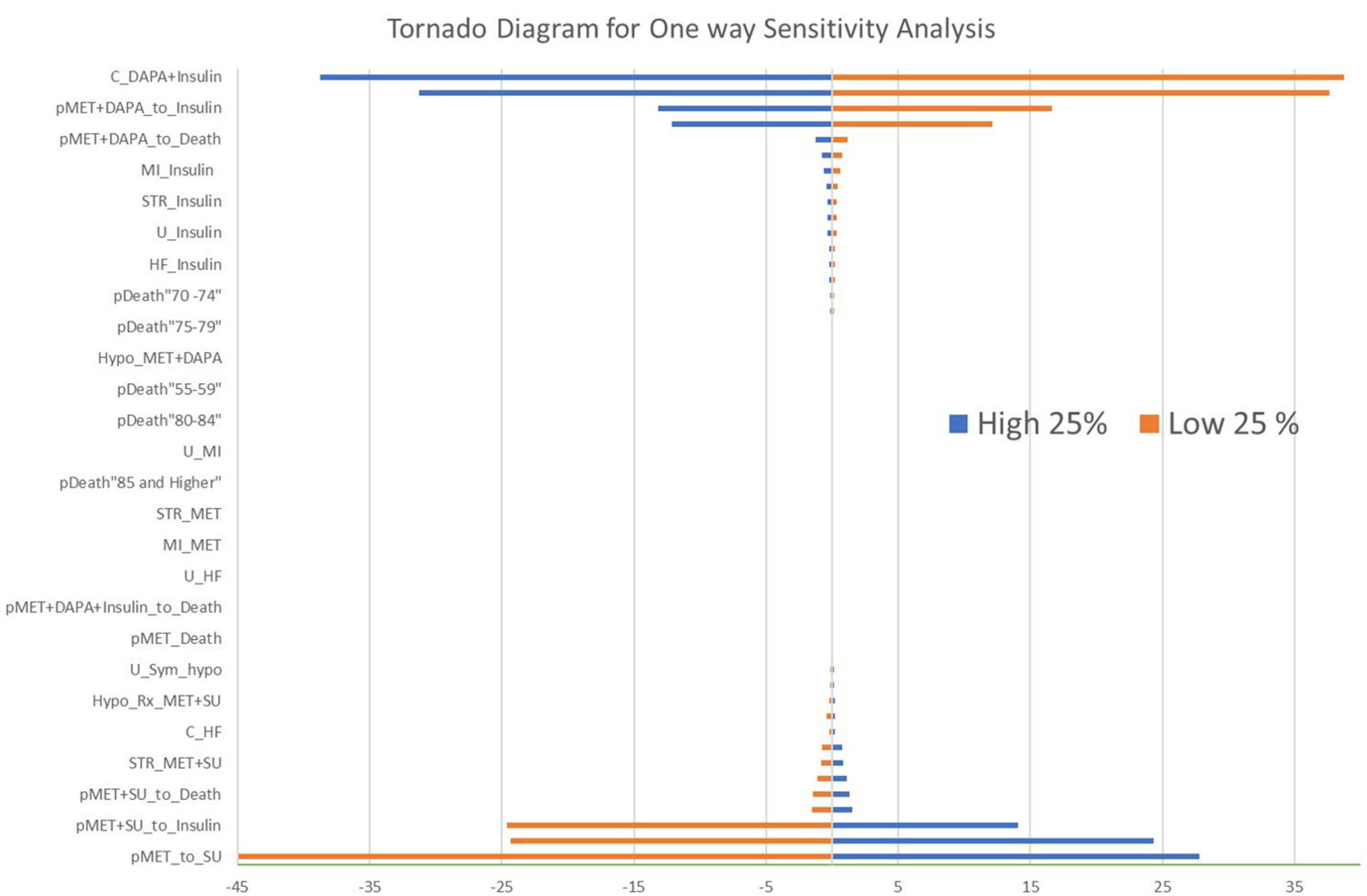

Figure 2 One-way sensitivity analysis. Author's compilation.

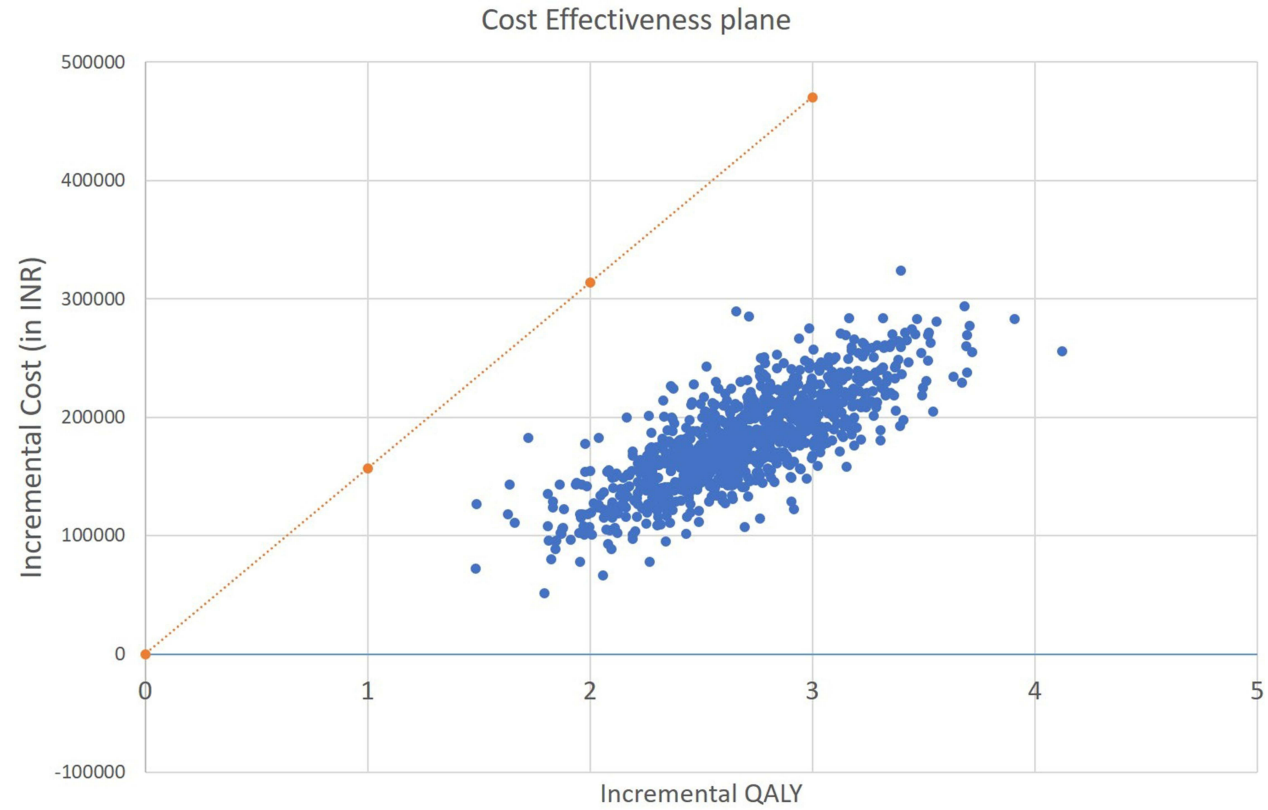

Figure 3 Cost-effectiveness plane. Author's compilation based on probabilistic sensitivity analysis. 


\section{Cost Effectiveness Acceptability Curve}

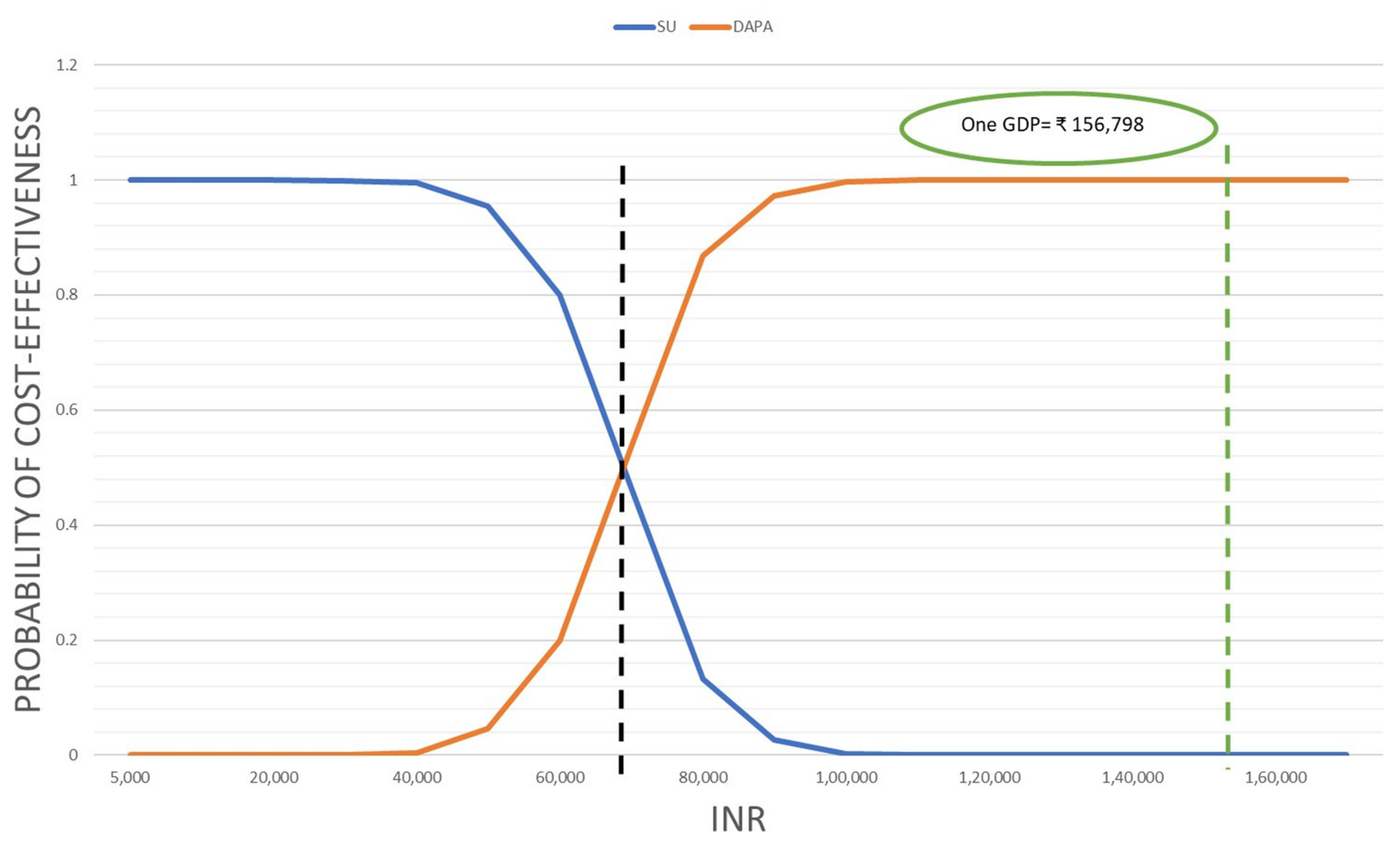

Figure 4 Cost-effectiveness acceptability curve.

could significantly decrease the risk for serious adverse events due to AKI events. ${ }^{31}$ Fourth, owing to limited data of head to head comparison between dapagliflozin and sulfonylureas in India, the efficacy data were retrieved from the Nauck et $\mathrm{al}^{21}$ study comparing dapagliflozin and glipizide. Nevertheless, we did not consider the class effect for different drugs in sulfonylureas. Therefore, future studies should further investigate such drug class effects. Last, we did not consider patients' direct non-medical costs, such as transportation and caregiver costs. Nevertheless, it is expected that dapagliflozin may be even more costeffective if direct non-medical costs are incorporated into the analysis, since dapagliflozin can better control the blood glucose level and reduce cardiovascular events compared to sulfonylureas.

In spite of these limitations, our study suggested that dapagliflozin would be cost-effective to be prescribed as second-line therapy compared to sulfonylureas for treatment of T2DM based on an Indian healthcare payer's perspective. This could provide the evidence-based information for physicians to make decisions on prescribing dapagliflozin as second-line therapy compared to sulfonylureas for
T2DM patients in India. Besides, the results from this study can also be used as the useful information for decision-makers in low- and middle-income countries.

\section{Ethics Approval and Consent to Participate}

It was exempted, as the secondary data were obtained from publicly available data sources.

\section{Declarations}

There is nothing to declare.

\section{Acknowledgments}

BSB acknowledges the financial fellowship supported by the Department of Health Research, Govt. of India through a fellowship under Human Resource development program, Indian Council of Medical Research. This work is a part of training in Health Technology Assessment (HTA)'s PhD/ Master degree, which scholarship is provided by Mahidol University and the International Decision Support Initiative (iDSI). This work was produced as part of the International Decision Support Initiative (www.idsihealth.org), which 
supports countries to get the best value for money from health spending. iDSI receives funding support from the Bill and Melinda Gates Foundation, the UK Department for International Development, and the Rockefeller Foundation. The findings, interpretations and conclusions expressed in this article do not necessarily reflect the views of the aforementioned funding agencies. This manuscript is an important part of the training of Dr. Bhavani Shankara Bagepally who is a Master's student in Health Technology Assessment in the Faculty of Pharmacy and Faculty of Graduate Studies, Mahidol University, Bangkok Thailand.

\section{Author Contributions}

All authors contributed to data analysis, drafting, or revising the article, have agreed on the journal to which the article will be submitted, gave final approval of the version to be published, and agree to be accountable for all aspects of the work.

\section{Funding}

BSB acknowledges the financial fellowship support by the Department of Health Research, the Government of India through a fellowship under Human Resource development program, Indian Council of Medical Research. This work is a part of training in Health Technology Assessment (HTA)'s Master degree, which scholarship is provided by Mahidol University and the International Decision Support Initiative (iDSI). This work was produced as part of the International Decision Support Initiative (www.idsihealth. org), which supports countries to get the best value for money from health spending. The iDSI receives funding support from the Bill and Melinda Gates Foundation, the UK Department for International Development, and the Rockefeller Foundation. The findings, interpretations and conclusions expressed in this article do not necessarily reflect the views of the aforementioned funding agencies.

\section{Disclosure}

BSB got funded for his master's course by the Department of Health Research, Government of India and International Decision support Initiative (iDSI). Funders has no role/ influence on the manuscript. The authors report no other conflicts of interest in this work.

\section{References}

1. IDF diabetes atlas - key messages. Available from: http://www.diabe tesatlas.org/key-messages.html. Accessed September 24, 2018.
2. Cho NH, Shaw JE, Karuranga S, et al. IDF diabetes atlas: global estimates of diabetes prevalence for 2017 and projections for 2045 . Diabetes Res Clin Pract. 2018;138:271-281. doi:10.1016/j. diabres.2018.02.023

3. Bagheri N, McRae I, Konings P, et al. Undiagnosed diabetes from cross-sectional GP practice data: an approach to identify communities with high likelihood of undiagnosed diabetes. BMJ Open. 2014;4(7): e005305. doi:10.1136/bmjopen-2014-005305

4. Beagley J, Guariguata L, Weil C, Motala AA. Global estimates of undiagnosed diabetes in adults. Diabetes Res Clin Pract. 2014;103 (2):150-160. doi:10.1016/j.diabres.2013.11.001

5. Fisher-Hoch SP, Vatcheva KP, Rahbar MH, McCormick JB, Kirchmair R. Undiagnosed diabetes and pre-diabetes in health disparities. PLoS One. 2015;10(7):7. doi:10.1371/journal.pone.0133135

6. India State-Level Disease Burden Initiative Collaborators. Nations within a nation: variations in epidemiological transition across the states of India, in the Global Burden of Disease Study. Lancet. 2017;390(10111):2437-2460. doi:10.1016/S01406736(17)32804-0

7. Anjana RM, Deepa M, Pradeepa R, et al. Prevalence of diabetes and prediabetes in 15 states of India: results from the ICMR-INDIAB population-based cross-sectional study. Lancet Diabetes Endocrinol. 2017;5(8):585-596. doi:10.1016/S2213-8587(17)30174-2

8. Oberoi S, Kansra P. Economic menace of diabetes in India: a systematic review. Int $J$ Diabetes Dev Ctries. 2020;1-12. doi:10.1007/s13410-020-00838-z

9. Bergman M, Buysschaert M, Schwarz PE, Albright A, Narayan KV, Yach D. Diabetes prevention: global health policy and perspectives from the ground. Diabetes Manag. 2012;2(4):309-321. doi:10.2217/dmt.12.34

10. Verma R, Khanna P, Mehta B. National programme on prevention and control of diabetes in India: need to focus. Australas Med J. 2012;5(6):310-315. doi:10.4066/AMJ.2012.1340

11. American Diabetes Association. 8. Pharmacologic approaches to glycemic treatment: standards of medical care in diabetes-2018. Diabetes Care. 2018;41(Suppl1):S73-S85. doi:10.2337/dc18S008

12. Azoulay L, Suissa S. Sulfonylureas and the risks of cardiovascular events and death: a methodological meta-regression analysis of the observational studies. Diabetes Care. 2017;40(5):706-714. doi: $10.2337 / \mathrm{dc} 16-1943$

13. Douros A, Dell'Aniello S, Yu OHY, Filion KB, Azoulay L, Suissa S. Sulfonylureas as second line drugs in type 2 diabetes and the risk of cardiovascular and hypoglycaemic events: population based cohort study. BMJ. 2018;362. doi:10.1136/bmj.k2693

14. Yesudian CAK, Grepstad M, Visintin E, Ferrario A. The economic burden of diabetes in India: a review of the literature. Glob Health. 2014;10:80. doi:10.1186/s12992-014-0080-x

15. Walker IF, Garbe F, Wright J, et al. The economic costs of cardiovascular disease, diabetes mellitus, and associated complications in South Asia: a systematic review. Value Health Reg Issues. 2017;15:12-26. doi:10.1016/j.vhri.2017.05.003

16. Bagepally BS, Gurav YK, Anothaisintawee T,et al. Cost Utility of Sodium-Glucose Cotransporter 2 Inhibitors in the Treatment of Metformin Monotherapy Failed Type 2 Diabetes Patients: A Systematic Review and Meta-Analysis. Value Health. 2019 Dec;22 (12):1458-1469. doi:10.1016/j.jval.2019.09.2750. Epub 2019 Nov 18. PMID: 31806203

17. Bertram MY, Lauer JA, De Joncheere K, Edejer T, Hutubessy R, Kieny MP, Hill SR. Cost-effectiveness thresholds: pros and cons. Bull World Health Organ. 2016 Dec 1;94(12):925-930. doi: 10.2471/BLT.15.164418. Epub 2016 Sep 19. PMID: 27994285; PMCID: PMC5153921.

18. NPCDCS program. National Programme for Prevention and Control of Cancer, Diabetes, Cardiovascular Diseases and Stroke (NPCDCS). Available from: https://dghs.gov.in/content/1363_3 NationalProgrammePreventionControl.aspx. Accessed November 26 , 2019. 
19. CPI data source. Available from: https://www.imf.org/external/pubs/ $\mathrm{ft} /$ weo/2018/01/weodata/download.aspx. Accessed November 26, 2019.

20. Unnikrishnan R, Anjana RM, Mohan V. Diabetes mellitus and its complications in India. Nat Rev Endocrinol. 2016;12(6):357-370. doi: 10.1038/nrendo.2016.53

21. Nauck MA, Del Prato S, Durán-García S, et al. Durability of glycaemic efficacy over 2 years with dapagliflozin versus glipizide as add-on therapies in patients whose type 2 diabetes mellitus is inadequately controlled with metformin. Diabetes Obes Metab. 2014;16 (11):1111-1120. doi:10.1111/dom.12327

22. NPPA ceiling price. Available from: http:/www.nppaindia.nic.in/en/ ceiling/press2june16/so1951e-2-6-16.html. Accessed March 26, 2018.

23. NPPA_1. Available from: http://www.nppaindia.nic.in/en/prno tif2014/pr-not-para19-2014.html. Accessed March 26, 2018.

24. Forxiga $10 \mathrm{mg}$ tab (AstraZeneca Pharma India Ltd). Available from: https://www.medplusmart.com/product/FORXIGA-10MG-TAB /FORX0002. Accessed March 26, 2018.

25. Wilding JPH, Woo V, Rohwedder K, Sugg J, Parikh S; Dapagliflozin 006 Study Group. Dapagliflozin in patients with type 2 diabetes receiving high doses of insulin: efficacy and safety over 2 years. Diabetes Obes Metab. 2014;16(2):124-136. doi:10.1111/dom.12187

26. Sosale B, Sosale A, Bhattacharyya A. Clinical effectiveness and impact on insulin therapy cost after addition of dapagliflozin to patients with uncontrolled type 2 diabetes. Diabetes Ther. 2016;7 (4):765-776. doi:10.1007/s13300-016-0204-9

27. Charokopou M, McEwan P, Lister S, et al. Cost-effectiveness of dapagliflozin versus DPP-4 inhibitors as an add-on to metformin in the treatment of type 2 diabetes mellitus from a UK healthcare system perspective. BMC Health Serv Res. 2015;15(1). doi:10.1186/ s12913-015-1139-y

28. Charokopou M, McEwan P, Lister S, et al. The cost-effectiveness of dapagliflozin versus sulfonylurea as an add-on to metformin in the treatment of type 2 diabetes mellitus. Diabet Med. 2015;32 (7):890-898. doi:10.1111/dme.12772

29. Sabale U, Ekman M, Granström O, Bergenheim K, McEwan P. Costeffectiveness of dapagliflozin (Forxiga ${ }^{\mathbb{R}}$ ) added to metformin compared with sulfonylurea added to metformin in type 2 diabetes in the Nordic countries. Prim Care Diabetes. 2015;9(1):39-47. doi:10.1016/j.pcd.2014.04.007

30. Tsapas A, Avgerinos I, Karagiannis T, et al. Comparative effectiveness of glucose-lowering drugs for type 2 diabetes: a systematic review and network meta-analysis. Ann Intern Med. 2020;173 (4):278-286. doi:10.7326/M20-0864

31. Menne J, Dumann E, Haller H, Schmidt BMW. Acute kidney injury and adverse renal events in patients receiving SGLT2-inhibitors: a systematic review and meta-analysis. PLoS Med. 2019;16(12): e1002983. doi:10.1371/journal.pmed.1002983

32. Kahn SE, Haffner SM, Heise MA, et al. Glycemic durability of rosiglitazone, metformin, or glyburide monotherapy. $N$ Engl J Med. 2006;355(23):2427-2443. doi:10.1056/NEJMoa066224

33. Mohan V, Shanthirani CS, Deepa M, Deepa R, Unnikrishnan RI, Datta M. Mortality rates due to diabetes in a selected urban south Indian population-the Chennai Urban Population Study [CUPS-16]. $J$ Assoc Physicians India. 2006;54:113-117.

34. Toulis KA, Willis BH, Marshall T, et al. All-cause mortality in patients with diabetes under treatment with dapagliflozin: a Population-Based, Open-Cohort Study in the health improvement network database. J Clin Endocrinol Metab. 2017;102 (5):1719-1725. doi:10.1210/jc.2016-3446

35. Varvaki Rados D, Catani Pinto L, Reck Remonti L, Bauermann Leitão C, Gross JL, Lehman R. The association between sulfonylurea use and all-cause and cardiovascular mortality: a meta-analysis with trial sequential analysis of randomized clinical trials. PLoS Med. 2016;13(4):e1001992. doi:10.1371/journal.pmed.1001992
36. Anyanwagu U, Mamza J, Donnelly R, Idris I. Comparison of cardiovascular and metabolic outcomes in people with type 2 diabetes on insulin versus non-insulin glucose-lowering therapies (GLTs): a systematic review and meta-analysis of clinical trials. Diabetes Res Clin Pract. 2016;121:69-85. doi:10.1016/j.diabres.2016.09.002

37. Mishriky BM, Cummings DM, Tanenberg RJ. The efficacy and safety of DPP4 inhibitors compared to sulfonylureas as add-on therapy to metformin in patients with type 2 diabetes: a systematic review and meta-analysis. Diabetes Res Clin Pract. 2015;109 (2):378-388. doi:10.1016/j.diabres.2015.05.025

38. Göke B, Gallwitz B, Eriksson J, Hellqvist A, Gause-Nilsson I; D1680C00001 Investigators. Saxagliptin is non-inferior to glipizide in patients with type 2 diabetes mellitus inadequately controlled on metformin alone: a 52-week randomised controlled trial. Int J Clin Pract. 2010;64 (12):1619-1631. doi:10.1111/j.1742-1241.2010.02510.x

39. Bosch J, Gerstein HC, et al; ORIGIN Trial Investigators. n-3 fatty acids and cardiovascular outcomes in patients with dysglycemia. $N$ Engl J Med. 2012;367(4):309-318. doi:10.1056/NEJMoa1203859

40. Kosiborod M, Birkeland KI, Cavender MA, et al. Rates of myocardial infarction and stroke in patients initiating treatment with SGLT2-inhibitors versus other glucose-lowering agents in real-world clinical practice: results from the CVD-REAL study. Diabetes Obes Metab. 2018;20 (8):1983-1987. doi:10.1111/dom.13299

41. Roumie CL, Greevy RA, Grijalva CG, et al. Association between intensification of metformin treatment with insulin vs sulfonylureas and cardiovascular events and all-cause mortality among patients with diabetes. JAMA. 2014;311(22):2288-2296. doi:10.1001/ jama.2014.4312

42. Gitt AK, Bramlage P, Binz C, Krekler M, Deeg E, Tschöpe D. Prognostic implications of DPP-4 inhibitor vs. sulfonylurea use on top of metformin in a real world setting - results of the 1 year follow-up of the prospective DiaRegis registry. Int J Clin Pract. 2013;67(10):1005-1014. doi:10.1111/ijcp.12179

43. Puckrin R, Saltiel M-P, Reynier P, Azoulay L, Yu OHY, Filion KB. SGLT-2 inhibitors and the risk of infections: a systematic review and meta-analysis of randomized controlled trials. Acta Diabetol. 2018;55 (5):503-514. doi:10.1007/s00592-018-1116-0

44. Clarke PM, Gray AM, Briggs A, et al. A model to estimate the lifetime health outcomes of patients with type 2 diabetes: the United Kingdom Prospective Diabetes Study (UKPDS) Outcomes Model (UKPDS no. 68). Diabetologia. 2004;47(10):1747-1759. doi:10.1007/s00125-004-1527-z

45. Currie CJ, Poole CD, Woehl A, et al. The health-related utility and health-related quality of life of hospital-treated subjects with type 1 or type 2 diabetes with particular reference to differing severity of peripheral neuropathy. Diabetologia. 2006;49(10):2272-2280. doi:10.1007/s00125-006-0380-7

46. Barry HC, Ebell MH, Hickner J. Evaluation of suspected urinary tract infection in ambulatory women: a cost-utility analysis of office-based strategies. J Fam Pract. 1997;44(1):49-60.

47. Zhang P, Brown MB, Bilik D, Ackermann RT, Li R, Herman WH. Health utility scores for people with type 2 diabetes in U.S. managed care health plans: results from Translating Research Into Action for Diabetes (TRIAD). Diabetes Care. 2012;35(11):2250-2256. doi:10.2337/dc11-2478

48. Actrapid HM Penfill 100IU 3ml INJ (ABBOTT) - buy actrapid HM Penfill 100IU 3ML INJ online at best price in India - MedPlusMart. Available from: https:/www.medplusmart.com/product/ACTRAPID-HM-PENFILL -100IU-3ML-INJ/ACTR0003. Accessed November 26, 2019.

49. Kwon CS, Seoane-Vazquez E, Rodriguez-Monguio R. Costeffectiveness analysis of metformin+dipeptidyl peptidase-4 inhibitors compared to metformin+sulfonylureas for treatment of type 2 diabetes. BMC Health Serv Res. 2018;18(1):78. doi:10.1186/ s12913-018-2860-0 
50. Gu S, Mu Y, Zhai S, Zeng Y, Zhen X, Dong H. Cost-effectiveness of dapagliflozin versus acarbose as a monotherapy in type 2 diabetes in China. PLoS One. 2016;11:11. doi:10.1371/journal.pone.0165629
51. Kwatra G, Kaur P, Toor G, et al. Cost of stroke from a tertiary center in northwest India. Neurol India. 2013;61(6):627-632. doi:10.4103/ 0028-3886.125270

\section{Publish your work in this journal}

ClinicoEconomics and Outcomes Research is an international, peerreviewed open-access journal focusing on Health Technology Assessment, Pharmacoeconomics and Outcomes Research in the areas of diagnosis, medical devices, and clinical, surgical and pharmacological intervention. The economic impact of health policy and health systems organization also constitute important areas of coverage. The manuscript management system is completely online and includes a very quick and fair peer-review system, which is all easy to use. Visit http://www.dovepress.com/testimonials.php to read real quotes from published authors. 